\title{
EI desarrollo del proceso de "Bolonia" y el Grado de Medicina. Situación actual y expectativas para su implantación definitiva
}

\author{
R.D. Lobato; A. Lagares; J.F. Alén y R. Alday
}

Servicio de Neurocirugía. Hospital "12 de Octubre”. Facultad de Medicina. Universidad Complutense. Madrid.

\section{Resumen}

La implantación del espacio europeo de educación superior (EEES) inspirado en la declaración de Bolonia conlleva la introducción de un nuevo paradigma docente que requiere reestructurar la enseñanza universitaria. Este proceso ha llevado a la reducción de la duración de los anteriores grados con excepción de unos pocos, entre los que se cuenta el nuevo Grado de Medicina. Aunque es un objetivo prioritario del EEES homogeneizar y armonizar las enseñanzas y las titulaciones en Europa para favorecer la circulación o intercambio de estudiantes y de profesionales, en el año 2010, cuando debería haberse completado el proceso boloñés, está muy lejos de conseguirse la deseada convergencia en la enseñanza superior en Europa.

En este artículo se comenta cómo el retardo en la armonización y la convergencia europea es especialmente relevante en los estudios de Medicina, y se analiza el desarrollo del proceso boloñés, tanto en Europa como en nuestro país, donde se están diseñando los nuevos Planes de Estudio de Medicina en un marco limitado por la improvisación burocrática y la precipitación derivadas de los plazos fijos impuestos por las autoridades políticas (límite en el 2010), la falta de financiación, y la inercia de sectores profesorales que desconocen lo esencial del proyecto y muestran indiferencia, o incluso resistencia, a cualquier cambio de paradigma docente. Así, y en contraste con lo ocurrido en otros países vecinos, en los que independientemente del proceso de Bolonia se han conseguido diferentes grados de modernización curricular, en España existe un alto riesgo de que los nuevos Planes se queden en meros cambios cosméticos de los utilizados previamente en la mayoría de las Facultades. De resultar así, la introducción de verdaderas innovaciones docentes, como las preconizadas desde el EEES, se vería seriamente limitada aumentando más aún la divergencia con Europa.

Recibido: 20-01-10. Aceptado: 26-02-10
PALABRAS CLAVE: Proceso de Bolonia. Espacio Europeo de Educación Superior. Currículum. Medicina. Enseñanza del pregrado.

Implementation of the Bologna system in medical education. Current status and future prospects

Summary

The implementation of the European Higher Education Area, (EEES in Spanish) inspired in the Bologna Declaration, pursues the introduction of new teaching and learning paradigms which require deep changes in the frame of superior education and university goals. However, in spite that the main purpose of the EEES is convergence and harmonization of curricula contents and titles throughout Europe in order to facilitate circulation of students and professionals, this goal is far from been reached when we are approaching the deadline for its implementation (year 2010). In addition, this process has led to reduce the total duration of the majority of degrees excepting for medicine and few more.

In this article we analyze the underdevelopment of the so called Bologna Process in medical education as compared to other careers. Implementation of curricular innovations seems particularly restrained or threatened in Spain because of legal improvisation, lack of funding, and the chronic apathy of national bodies in medical education. As a consequence, and in contrast with other European countries where deep curricular changes have been already arranged, the majority of Spanish Faculties are at risk of introducing little more than cosmetic modifications in their medicine curricula.

KEY WORDS: Bologna system. European Higher Education Area. Medical currículum. Undergraduate teaching.

Introducción

El llamado proceso de Bolonia persigue desarrollar 
la Declaración de Bolonia de 1999 para crear un Espacio Europeo de Educación Superior (EEES o EHEA en inglés i.e., European Higher Education Area) mediante el logro de los siguientes objetivos: 1 . Reforzar la competitividad de la educación superior europea en el ámbito internacional; 2 . Favorecer la "empleabilidad" y movilidad de los ciudadanos europeos; 3 . Adoptar un sistema simple y comparable de grados; 4. Implantar un sistema de enseñanza basado en tres ciclos (Grado, Master y Doctorado); 5. Establecer un sistema de medida con nuevos créditos docentes (los ECTS del "European Credit Transfer System"); 6. Promocionar la movilidad de estudiantes y profesores entre países superando los obstáculos existentes; 7. Propiciar la cooperación en la certificación de la calidad de los procesos docentes; y como objetivo más general, 8. Promocionar el sistema de la educación superior en Europa ${ }^{5}$.

A estos objetivos, que deberían ser alcanzados respetando siempre la diversidad de culturas y lenguas, así como las características de los sistemas educativos de las diversas naciones y la autonomía de sus universidades, se añadieron en las reuniones de Praga (2001) y Berlín (2003) los de: 9. Integrar el aprendizaje de por vida en la estrategia educativa global; 10. Implicar a los estudiantes en el desarrollo del proceso; 11. Promover mediante una estrategia de información apropiada el atractivo del EEES, y, 12. Crear el Area Europea en Investigación. Después se han mantenido reuniones bianuales en Bergen (2005), Londres (2007), Lovaina (2009), Budapest-Viena (2010), habiéndose programando la próxima en Bucarest (2012) y otras en lugares por determinar para los años 2015, 2018 y el 2020, cuando el proceso deberá haberse completado en todos sus apartados. Al margen de estas reuniones de carácter "institucional" paneuropeo, se han celebrado numerosos workshops, seminarios y otros encuentros de ámbito nacional o internacional.

Antes de abordar el objetivo concreto de este escrito, conviene hacer notar que el desarrollo del proceso boloñés arrancó y ha sido conducido sin una estructura organizativa sólida, una fuerza impulsora centralizada, un mecanismo o estrategia para resolver las posibles disputas, y un soporte financiero adecuado, por lo que se ha llegado a decir que en realidad es un "unmanaged process" ha estado dirigido desde estamentos políticos que han creado numerosos cuerpos burocráticos y agencias de gestión en los que no han sido adecuadamente integrados los agentes implicados pertenecientes a las universidades, lo cual podría explicar, al menos en parte, la llamativa falta de crítica desde las instituciones académicas y las reticencias mostradas por amplios sectores de alumnos y profesores y grupos de expertos en educación, así como los actuales desajustes en su implementación, y la incertidumbre sobre su culminación exitosa.

La Asociación Europea de Estudiantes ha manifes- tado su frustración al quedar excluida de este desarrollo, culpando de engaño e incumplimiento de promesas a los responsables políticos. Igualmente, la influencia del profesorado como colectivo en la definición de los objetivos y la participación en las decisiones ha sido marginal, y es que las convocatorias y reuniones preparatorias de las agendas de trabajo se han hecho casi siempre a través de "contactos" con personal "civil" que trabajan para los Ministerios de Educación de los diferentes países, lo cual ha propiciado malestar y pasividad de los sectores académicos. Como señala Christensen ${ }^{3}$, las tímidas llamadas para una mayor participación de estos sectores no han llegado desde las universidades, sino desde los cuarteles de ese personal "civil" (e.g. la Salamanca Convention y Reunión de Praga del 2001, o la Declaración de Graz del 2003).

No es el propósito de este análisis entrar en el trasfondo del debate político y filosófico sobre el ambicioso proyecto de creación de un "espacio común de educación superior", ni atizar la polémica entre quienes acusan a la universidad de querer perpetuarse en la "provincialización" y el localismo, oponiéndose así a la "internacionalización", y aquellos otros que nos advierten del riesgo de su "instrumentalización" por fuerzas ajenas a la misma. Sin embargo, conviene dejar claro que considerar las críticas al proceso de Bolonia como "rancios posicionamientos de izquierdas" es una burda simplificación. $\mathrm{Y}$ es que para muchos este proceso se inserta en un neoconservadurismo que podría instrumentalizar la universidad hasta acabar con su autonomía, de tal manera que, al albur de Bolonia, la Europa que creó la Universidad hace casi ocho siglos, podría ahora hacerla desaparecer entregándola sin condiciones a los intereses de la gran patronal y las fuerzas del mercado ${ }^{4,8,11}$.

No cabe duda que la universidad haría dejación de sus principales funciones si se inclinara preferentemente hacia la "profesionalización", remodelando a la baja los grados a satisfacción de demandas "empresarias" en todas las disciplinas, incluyendo las humanidades, que a pesar de haber estado en la base de la génesis de la institución universitaria han quedado ahora lamentablemente marginadas por el Plan de Bolonia ${ }^{8}$. Böhm nos advierte que cuando la investigación tiene que legitimarse demostrando continuamente su relevancia social se acaba con el deseo espontáneo de conocer y saber, que es el motor de la investigación pura y una de las tres funciones cardinales de la institución universitaria. Una "instrumentalización" de este cuño, patrocinada no ya por la Iglesia o el Estado como ocurrió en el pasado, sino por las fuerzas que rigen el mercado (¿"mercantilización"?), la convertiría en una institución inerme e incapaz de defenderse a sí misma, que tras perder su autonomía, no ostentaría el debido liderazgo en la conformación del futuro de la sociedad a la que pertenece y se debe.

Abraham Flexner, que reformó la enseñanza de medi- 
cina a principios del siglo XX basando su nuevo paradigma docente sobre una universidad creadora y transmisora de ciencia más que sobre otra "fabricante" de profesionales, apunta en su libro Universities: American, English, German: "Intellectual inquiry -not job training- is the purpose of the university", y en la celebración de su centenario la medicina académica norteamericana trata de seguir siendo fiel a los mismos presupuestos?

En su obra El mito de la autonomía universitaria, Sosa Wagner $^{11}$ escribe lo que sigue acerca de una universidad "que asuma los modos y maneras de la empresa, y que cultive su lógica y su logística": Este proceso que no ha hecho sino comenzar, sólo espera la brisa benéfica del tiempo y los afanes sostenidos e incansables de los funcionarios europeos. Decenas de reuniones de ministros, de altos funcionarios, cientos de seminarios, de declaraciones, de acuerdos, de documentos, de agotadoras comidas de trabajo, miles de viajes de vicerrectores de la ceca a la meca, irán jalonando el periodo histórico que ahora abre los ventanales y se asoma al futuro. Pues bien, la autonomía de la universidad tiene en este punto fecha de caducidad...

Pero independientemente del riesgo añadido que la implantación de Bolonia pueda suponer para la autonomía universitaria, en nuestro país las universidades han pervertido ya en gran manera el espíritu y la letra de la Ley de Autonomía Universitaria, que bajo la bandera de la libertad de cátedra y de expresión consagra a la institución universitaria como bastión de la educación y la cultura. Según recoge en su artículo primero, esta Ley confía a los profesores y gestores universitarios, a quienes entroniza en la cúspide de los valores intelectuales y éticos, el futuro de la educación superior. Sin embargo, muchos de ellos parecen ignorar el penúltimo punto del artículo que dice "la autonomía universitaria exige y hace posible que docentes, investigadores y estudiantes cumplan con sus respectivas responsabilidades, en orden a la satisfacción de las necesidades educativas, científicas y profesionales de la sociedad, así como que las universidades rindan cuentas del uso de sus medios y recursos a la sociedad".

Desafortunadamente, en la mayoría de nuestras universidades, presas desde hace mucho tiempo de un corporativismo casi letal, la distorsión del concepto y el uso de la "libertad de cátedra" supone una limitación para implantar cualquier paradigma docente nuevo, sea o no el que se preconiza desde el EEES (este paradigma docente que no ha sido "creado", sino adoptado por los cuerpos burocráticos que conducen el desarrollo del EEES, y que ha sido incorporado ya en numerosos curricula durante las dos-tres últimas décadas, conlleva: I. Una mayor implicación y autonomía del estudiante; II. La utilización de metodologías más activas: práctica clínica, trabajo en equipo, tutorías, tecnologías multimedia...; y III. Un cambio en el papel del profesorado, que actuará como agente creador de entornos de aprendizaje que estimulen a los alumnos, y no como mero transmisor de información).

En el apartado final de este escrito volvemos sobre el ejercicio de la "libertad de cátedra" ya que se trata de un punto esencial en la mejora de cualquier programa docente. Pero pasemos a comentar cual es el estado actual de la implementación del proceso de Bolonia en los nuevos Grados de Medicina.

\section{“Bolonia y Medicina”}

De entrada conviene hacer notar que la peculiar estructura de la carrera de medicina la protege de asechanzas que de la mano de Bolonia han menoscabado ya otros grados. En primer lugar, y sin saber bien con qué criterios, unas pocas carreras entre las que están Medicina y Arquitectura han sido definidas como "profesiones de especial o mayor interés social", por lo que sus grados no han sufrido la amputación que ha afectado a la gran mayoría de las antiguas licenciaturas convertidas ahora en grados de 4 años (240 ECTS); en segundo lugar, la "enseñanza por competencias", que puede resultar problemática, o incluso no aplicable en otros grados, parece de ineludible incorporación en medicina si queremos que el nuevo graduado adquiera no sólo los conocimientos necesarios, sino también las habilidades y actitudes que le capaciten para la buena práctica clínica; en tercer lugar, al superar los 300 ECTS, el graduado en medicina no tendrá que realizar obligatoriamente un master para acceder al doctorado; y en cuarto lugar, la existencia de una formación de postgrado de buena calidad y carácter universal en Europa (sistema de residencia), junto con el hecho de que la "empleabilidad" del médico depende aún mayoritariamente de los sistemas nacionales de salud (empleador único o universal), protege al graduado en medicina de agravios que sí sufrirán otros, cuya formación, degradada o descafeinada, deberá "completarse" con masters de elevadísimos costes que les coloquen en cabeza a la hora de acceder al empleo en el mundo de la empresa privada.

Sin embargo, no todo queda allanado en este sentido, pues la tendencia a la privatización de la gestión de la asistencia sanitaria, podría cambiar tanto las condiciones de contratación del médico, como la calidad de la propia enseñanza del pregrado y del postgrado. Por otra parte, en nuestro país, el grado en medicina se denominará Graduado en Ciencias de la Salud, es decir, igual que otros que se adquieren cursando tan solo 240 ECTS (como el de Enfermería); por ello, la Confederación Estatal de Sindicatos Médicos (CEMS) (ver Diario Médico 17-VI-2009,) la Conferencia Nacional de Decanos y el Consejo Estatal de Estudiantes de Medicina, denuncian lo que consideran un agravio comparativo de Bolonia al médico, y además de reclamar que en los 360 ECTS del grado de medicina se 
incluya el master, exigen que se mantenga la denominación de "médico" para el nuevo graduado.

\section{El desarrollo del proceso de Bolonia en los estudios de Medicina}

Para abordar el objetivo de este artículo nos preguntaremos ¿Cómo se ha desarrollado hasta la fecha el proceso de Bolonia en relación con la enseñanza de la medicina a nivel europeo, y más concretamente en nuestro país? ¿Cual es el nivel de aceptación y adherencia actual al esquema "boloñés" en los diversos países? ¿Es ineludible ceñirse al mismo para modernizar el curriculum de medicina, o esto puede hacerse al margen del mismo, como se ha demostrado en otros países? ¿Es posible alcanzar la convergencia europea en los estudios de medicina, o se generará más bien una divergencia que dificultará la pretendida armonización de grados, la homologación de los títulos, la "libre circulación" de profesores y alumnos, y la implantación de los proyectados grados compartidos" ("joint degrees")? ¿Se está consensuando un "lenguaje común en terminología docente", o se está creando más bien una "torre de Babel" en este apartado? ¿Sabemos ya con exactitud que hay detrás de un ECTS? ¿Resultaría aplicable y beneficioso en términos de convergencia instaurar exámenes de licenciatura nacionales con un diseño homogéneo, o incluso un examen paneuropeo similar al realizado en USA y Canadá? y finalmente, y remitiéndonos al ámbito de nuestro país, ¿Seremos capaces de modernizar los programas docentes en nuestras Facultades para "coger el paso" de los países vecinos que han actualizado ya sus curricula independientemente del proceso boloñés, o por el contrario, y como ha ocurrido otras veces en el pasado, representaremos una excepción más o menos afrentosa en "el concierto de las naciones"?

Por razones poco claras, y dentro de la tónica de falta de participación de la universidad en este proceso, el retardo en el desarrollo del nuevo grado de medicina ha resultado mayor que en todos los demás. Durante años, la medicina ha estado ausente en las discusiones de los encuentros bianuales, y resulta casi increíble que en la enorme cantidad de papel generado en ellas no existiera hasta hace poco ninguna mención sobre los estudios de medicina, ni propuestas provenientes de escuelas, sociedades y organizaciones médicas. Como dice Christensen ${ }^{3}$, en contraste por ejemplo con las ingenierías, la arquitectura, las filologías o la informática, las brevísimas menciones a la medicina siempre aparecían entre paréntesis, de tal modo que se ha llegado a hablar de la medicina como "the subject in parentheses"; y de ahí afirmaciones tales como "el mantenimiento de un solo ciclo en un número muy limitado de grados, como por ejemplo medicina, no contraviene el proceso de Bolonia"; o, "al día de la fecha hay cientos de nuevos "bachelors" y "masters" cubriendo todas las áreas, excepto en medicina y teología"; y más recientemente "la medicina y algunas otras disciplinas requerirán todavía esquemas diferente en muchos países, con programas integrados de 300 o incluso más ECTS".

A la postre se considera a la medicina como el "último recién llegado" ("Medicine is the latecomer"), y esta tardanza en la "llegada" resulta de especial riesgo en los países "perezosos" como el nuestro, donde sin haberse realizado intentos de modernización curricular durante las últimas décadas, hay ahora que diseñar "a toda prisa" (límite en el 2010) los nuevos Planes de Estudios, que podrían quedarse así en meros maquillajes de los actualmente vigentes.

El estado actual del proceso de convergencia europea en los estudios de Medicina

Diferentes análisis recientes confirman una preocupante lentitud y dispersión en el desarrollo del proceso de convergencia en los estudios de Medicina ${ }^{3,10}$. En efecto, sólo una minoría de países han optado por el esquema "boloñés" de grado de 6 años de duración y 360 ECTS, que incluye un ciclo de Bachelor (180 ECTS) y otro de Master (180 ECTS), seguido de un master de postgrado, y finalmente de los estudios de doctorado. La realidad es que en el 2009 el proceso de convergencia en Medicina estaba pobremente desarrollado, y muchos analistas consideraban que había ya más divergencia que convergencia a lo largo y ancho de Europa.

Con objeto de conocer la situación real del proceso "boloñés" la AMEE (Association for Medical Education in Europe) y MEDINE (The Thematic Network on Medical Education in Europe) realizaron en el 2007 una encuesta dirigida en principio a las autoridades responsables (ministeriales) de 46 países implicados (algunos de ellos todavía no son miembros de la CEE). Las preguntas fueron: 1. ¿Qué estructura tiene el grado actualmente en su país? y 2. ¿Qué decisión se ha tomado en su país acerca de la adopción del sistema de dos ciclos (Bachelor + Master) para el grado de medicina propuesto en la Declaración de Bolonia? ¿Y en caso de haberse adoptado tal esquema, cuál es el nivel de desarrollo que se ha alcanzado en su implantación?

Los resultados del estudio publicados hace poco menos de un año ${ }^{10}$, indican que: A. Se registra una inesperada y llamativa lentitud y negligencia generalizada en las respuestas, que casi siempre fueron aportadas por personas "relacionadas" con las autoridades oficiales de cada país, pero no directamente por éstas, a pesar de lo cual se computaron como válidas; $\mathrm{B}$. Se comprobó que en la mayoría de los casos los educadores están desconectados en gran manera del proceso de convergencia, desconociendo cual es la situación real del mismo en sus propios países; 
además apenas se aportan "respuestas oficiales" acerca de las decisiones legislativas tomadas sobre la adopción o rechazo del Plan de Bolonia (lo cual reproduce lo ocurrido en otros grados y resulta sumamente preocupante); C. En la actualidad, la duración del grado es de 6 años en la mayoría de los países, excepto en el Reino Unido y Malta donde es de sólo 5 años; en Suecia dura 5.5 años (incluye sólo 330 créditos ECTS en el nuevo currículum introducido en el 2007), y en Bélgica y Alemania se toma 7 años; D. Un total de 19 países (46\%) responden que no van a adoptar la estructura de dos ciclos, y sólo 7 países (17\%), que son Armenia, Holanda, Portugal, Islandia, Bélgica, Dinamarca y Suiza la han adoptado; 4 países $(9 \%)$ dejan el asunto a elección de sus Facultades, y 11 (27\%) no han tomado todavía ninguna decisión al respecto, si bien se prevé que la mayoría de estos últimos rechazarán el grado con los dos ciclos de Bachelor y Master.

Por otra parte, expertos en educación médica participantes en diferentes reuniones sobre el proceso de Bolonia (e.g. Workshop en Bruselas en Mayo del $2005^{13}$, y otros posteriores), han señalado también las graves indefiniciones y el peligroso retardo en los acuerdos sobre la armonización y el proceso de convergencia, advirtiendo que existen: A. Modalidades de implantación de los ciclos Bachelor + Master con duraciones diferentes y contenidos curriculares y distribución de créditos muy variables que dificultarán la circulación del estudiante no ya en Europa, sino dentro de un mismo país (Bélgica por ejemplo); B. Reservas sobre el riesgo que supone la mercantilización y la intromisión de la industria y las compañías y empresas en la autonomía de las universidades en aras de la implantación de la convergencia, que están en la base de las manifestaciones de contrariedad de aquellos sectores estudiantiles y del profesorado que ha demostrado un mayor interés y conocimiento acerca de qué es realmente el proceso de Bolonia; C. Riesgos de deformación del concepto de crédito europeo hacia otro de puntuaciones (existen ya ejemplos), y D. La existencia de un pesimismo generalizado derivado de la falta de transparencia y de participación estructurada de todos los agentes implicados.

Por ello, se vienen sugiriendo alternativas "armonizadoras" como son las alianzas entre universidades concretas para facilitar la circulación de sus masters (e.g entre Leiden y el Karolinska), o el desarrollo de un sistema común de evaluación europeo (European Consortium for Accreditation creado en 2003). Además, son muchos quienes reclaman aclarar con urgencia las terminologías usadas a lo largo y ancho de Europa en relación con los grados y títulos, que el proceso de Bolonia no sólo no ha homogeneizado, sino que contribuye a confundir. En todo caso, es claro que estas iniciativas traslucen una seria disfunción en la implantación del EEES, y que la situación actual en relación con el proceso de convergencia en los estudios de medicina en Europa es cuando menos confusa e intranquilizadora.

Las conclusiones de estos análisis podrían ser:

1. Pocos docentes están bien informados acerca del proceso de la convergencia europea en la enseñanza de la medicina.

2. La división en dos ciclos se ha implantado en casi todas las carreras y en casi todos los países (grados de 4 años con 240 ECTS), excepto en el caso de Medicina ("la disciplina entre paréntesis").

3. Hay un consenso generalizado en que la implementación de los dos ciclos (Bachelor y Master) debe ser rechazada en Medicina porque atenta contra la integración de las enseñanzas básicas y clínicas, que se contempla ya en diferentes curricula, y es de trascendental relevancia para una buena enseñanza; en todo caso, si se adoptara un grado con dos ciclos, no debería permitirse que ello consolide o perpetúe la división entre las ciencias básicas y clínicas presente en los curricula "tradicionales", como los de las Facultades españolas, y que fue ya denostada por Flexner en su informe seminal sobre la docencia de la medicina de 1910; además, la exposición precoz al medio clínico del estudiante, necesaria para su formación como médico, resultaría superflua para los estudiantes que tras el Bachelor deriven su carrera hacia otras vías o profesiones. La educación médica es larga y cara, por lo que permitir, o incluso favorecer, su abandono representa un despilfarro de recursos; y es que el "bachelor degree" tiene muy poco valor para quienes no vayan a completar el grado y se desvíen hacia otras opciones profesionales a las que podrían acceder de otra manera más directa (e.g. biología, salud pública, odontología, o empleos en la industria farmacéutica).

4. Sin un acuerdo o consenso europeo en relación con la implementación del sistema de dos ciclos se puede dañar seriamente la comparabilidad de los títulos y la movilidad de los estudiantes y graduados. En los nuevos curricula de algunos países se enseñan materias a lo largo de varios años, por lo que la adopción de los dos ciclos conllevaría una separación artificial de esas materias o módulos, menoscabando la calidad de la enseñanza que se imparte actualmente; esta limitación sólo se podría obviar introduciendo unas guías claras para el Bachelor y el Master, como se hace en el European Core Curriculum en educación medica mencionado por el British General Medical Council en 1993, y definido después en la guía $n^{0} 5$ de la AMEE.

5. Aunque se ha aventurado que la aplicación de los ECTS, esencial para garantizar la comparabilidad de los títulos, no planteará problemas diferentes en medicina en comparación con otros grados, es muy probable que no sea así porque la aplicación de este sistema de medida requiere, más que en ninguna otra licenciatura o grado, de paquetes 
informativos que incluyan una descripción precisa de la unidad del currículum en la disciplina de que se trate, y que expliciten sus contenidos exactos, el nivel en el que se aplican y los métodos docentes y de evaluación a utilizar. $\mathrm{Y}$ es que para lograr comparabilidad y compatibilidad, y "converger" haciendo finalmente intercambiables los títulos sin agravios entre países que quieren intercambiar sus profesionales, no basta con "suscribir" la aceptación de los estándares internacionales de calidad en la enseñanza, o usar los mismos términos (e.g. bachelor y master), sino que es imprescindible predefinir claramente las competencias o resultados a conseguir en cada etapa del grado; pues bien, este objetivo no ha sido alcanzado, por lo que tienen razón quienes afirman que todavía nadie sabe bien qué hay detrás de un ECTS (un crédito ECTS -abreviación de European Credit Transfer System- equivale a 25 horas de trabajo del estudiante en el que se incluyen no sólo las horas lectivas y de prácticas, sino las empleadas en el estudio personal; un curso académico rinde 60 créditos ECTS).

6. Del mismo modo, a pesar de que la movilidad es un objetivo común a todos los niveles de los estudios, desde los cursos individuales y las rotaciones clínicas (incluyendo las del programa Erasmus), hasta los grados completos, no se han adoptado todavía guías para determinar unos contenidos comunes.

7. Igualmente, aunque se considera esencial un acuerdo y cooperación sobre sistemas que garanticen el control de la calidad de las enseñanzas, éstos no se han desarrollado, y se elude encargar su diseño y aplicación a cuerpos de expertos independientes como podrían ser los pertenecientes a la AMEE, que asumirían la tarea con total garantía. Si bien la adopción de estándares comunes de calidad, como los WFME Global Standards for Quality Improvement, permitiría alcanzar el objetivo de un sistema común de acreditación de escuelas de medicina, y proporcionaría un instrumento valioso para mantener una calidad docente alta y comparar con fiabilidad los rendimientos de las diferentes escuelas o facultades, este objetivo tampoco está contemplado de manera programática.

8. Al día de hoy existe una marcada confusión en las definiciones o términos usados en relación con la enseñanza de la medicina, que la Declaración de Bolonia no sólo no ha aclarado, sino que acentúa cada vez más; así, el propio término de "undergraduate", que en toda la literatura sobre docencia médica se usa habitualmente para referirse a los 5-6 años de los actuales grados, en el proceso boloñés se reserva para el Bachelor (el primer ciclo de los estudios de 3 años de duración). Términos como Bachelor y Doctor (Physician vs $\mathrm{PhD}$ ) se emplean también con diferentes significados; por ejemplo, en los 7 países que se han adherido a la Declaración adoptando el grado con los dos ciclos de 3 años, el término Bachelor recibe diferentes denominaciones, tales como Bachelor a secas, Bachelor of
Science en Islandia, Bachelor in Medicine en Suiza y Dinamarca, y Licenciado en Ciencias de la Salud en Portugal; como positivo hay que destacar que la naturaleza de este primer ciclo en esos países se define de manera homogénea como exclusivamente académica (no permite el ejercicio profesional).

Del mismo modo, la naturaleza o acreditación para la práctica profesional que concede el segundo ciclo o Master (180 ECTS) tampoco es totalmente homogénea, y así varía desde la exclusivamente académica (no permite la práctica profesional) como en Islandia, a una Profesional pura (permite la práctica) como en Bélgica, o Académico-Profesional como en los restantes 5 países; además, su denominación exacta también es diversa, e.g. Master Degree en Bélgica, Master in Medicine en Portugal y Suiza, y Master of Science in Medicine and Arts Diploma en Holanda.

La misma dispersión se observa en el nivel de exigencia de la realización de un master tras el grado para acceder al doctorado. Ya apuntamos que la carrera de medicina tiene la peculiaridad de que el grado va seguido de un sistema de formación especializada que nadie prevé por el momento sustituir por otro mejor. Así, el graduado en medicina, que si se dedica plenamente a su tarea de residente, no dispone de tiempo para realizar uno o más masters de postgrado, podrá obtener la preparación y orientación necesarias para realizar la Tesis doctoral durante la residencia. Por ello, es un acierto no exigir ni la realización de un master, ni la de los viejos "cursos" preparatorios del doctorado que tan caros e inútiles resultaban. En todo caso, la situación en este apartado diferirá también en España respecto a otros países vecinos, lo que contribuirá a aumentar la divergencia ya existente, que se propagará así del pregrado al postgrado, complicando la homologación de los perfiles de los médicos formados en ellos.

Por tanto, parece que en los estudios de Medicina se está lejos de alcanzar la ideal homogeneidad o armonización auspiciada por la Declaración de Bolonia, lo cual no excluye que pueda ser finalmente conseguida. La implantación de un examen final de grado a nivel paneuropeo podría quizás facilitar el camino hacia la convergencia, pero lo que parece muy difícil es que en aras de la armonización, los países que han desarrollado ya nuevos curricula, los remodelen para alcanzar un común denominador en el proceso de la evaluación que muchos no consideran ni siquiera deseable (ver más abajo).

\section{La implantación de exámenes finales de grado de ámbito nacional o paneuropeo como refuerzo del proceso de con- vergencia y la comparabilidad de títulos}

En un reciente encuentro mantenido en Praga, grupos de expertos en evaluación del aprendizaje clínico han debatido la pertinencia de aplicar un examen de final de 
grado en Europa, que, al menos en principio, propiciaría la armonización de los curricula, la homologación de títulos y la movilidad de estudiantes y profesionales ${ }^{1,6,9,12}$. Este examen podría ser similar al USMLE (United States Medical Licensing Examination) aplicado en USA o Canadá que en sus tres pasos o "steps" administrados en diferentes momentos del grado, evalúa además de los conocimientos teóricos del alumno, el nivel de competencia al aplicar éstos a la resolución de problemas clínicos utilizando para ello pacientes estandarizados y casos presentados en ordenador con formatos innovadores (www.usmle.org y www.mcc.ca; Guide to Good Medical Practice - USA. www.gmp-usa.org.).

Según la opinión de varios expertos, implantar exámenes tipo USLME, bien a nivel nacional, o bien a nivel paneuropeo, ayudaría a: 1. Homogenizar el nivel de competencia de los graduados egresados desde las diferentes escuelas de medicina europeas; 2 . Mejorar la convergencia de los curricula, la comparabilidad de los títulos y la movilidad de estudiantes y profesores; 3. Mejorar la asistencia al paciente en el contexto de la libre circulación de profesionales; 4. Garantizar el control de calidad de los estándares de las cada vez más numerosas escuelas privadas de medicina; y 5. Controlar el nivel de competencia y las habilidades psicosociales de graduados internacionales (profesionales no europeos) que acceden a la práctica en nuestros sistemas de salud ${ }^{1}$.

Además, un examen paneuropeo favorecería la aplicación de auditorías externas que reforzarían el cumplimiento de los estándares docentes y la transparencia y fiabilidad de la prueba, impulsaría la innovación curricular al evidenciar el rendimiento de los estudiantes y las deficiencias en su formación, y propiciaría una mejora progresiva del propio proceso de evaluación. Finalmente, la agregación de los esfuerzos de todas las escuelas y facultades en la configuración y aplicación de esa prueba única, disminuiría los costes de los exámenes múltiples de ámbito nacional, y permitiría aprovechar mejor las prestaciones de los grupos de expertos en evaluación que ahora trabajan dispersos en Europa. Sin embargo, otros expertos igualmente acreditados o incluso con mayor prestigio, nos advierten que aparte de las dificultades de tipo logístico que tendría la realización de un examen paneuropeo, existen serios argumentos en contra de su implantación.

Así, Cees van der Vleuten ${ }^{12}$ y Ronald Harden 6 , se preguntan si este tipo de examen no representará en realidad un impedimento para emprender cualquier cambio educativo o curricular en vez de favorecerlo. Para éstos y otros muchos expertos en educación médica es axiomático que la evaluación dirige el aprendizaje y transforma, mejorándolo, cualquier programa docente ("assessment drives learning" and "assessment enhances teaching"), y la literatura médica reciente nos demuestra que se está produciendo un desplazamiento de la cultura de pasar tests (evaluación sumativa para determinar si el candidato pasa/no pasa), a la cultura de evaluar para formar (evaluación formativa); la primera se aplica en momentos fijos a lo largo del grado, en tanto que la segunda se engrana con el propio aprendizaje en el contexto clínico real, al cual propulsa continuamente. Y es que el "assessment of learning" está dejando paso al "assessment for learning", (evaluación con retroalimentación inmediata), y está claro que los exámenes de licenciatura separan o alejan la evaluación del propio proceso del aprendizaje (del "ongoing learning").

De acuerdo con estos autores, los exámenes finales de licenciatura, sean del tipo USMLE, que incluye pruebas prácticas con enfermos estandarizados, o del tipo del que se pasa en Francia o nuestro examen MIR que no las incluyen, sólo permiten evaluar habilidades y competencias "fáciles de medir" ("low profile tasks"), pero no las complejas, con lo cual su validez o utilidad es tan sólo limitada, independientemente de cuales sean su practicabilidad y costes. Esta clase de exámenes llamados "single shot assessment" acarrearían, por un lado, una armonización reduccionista de curricula modernizados de eficacia ya probada, como los que están operativos en muchas escuelas del Reino Unido y de otros países del centro y norte de Europa que incluyen estrategias de evaluación objetiva estructurada imbricadas con el proceso del aprendizaje clínico continuo; además comprometerían, por otro lado, la diversidad ("pluriformity") de los programas docentes, que es una de las connotaciones de la enseñanza europea que, según el propio Plan de Bolonia, debe ser preservada y respetada a toda costa.

Ronald Harden 6 "desmonta" lo que llama los 5 mitos a favor del examen paneuropeo. Así, y en relación con el primero de los mitos, niega que un examen centralizado (tanto a nivel nacional, como transnacional) asegure la evaluación del nivel de competencia global de los candidatos, que es en definitiva lo que importa; otra vez más argumenta que tal examen sólo evaluaría las llamadas "low level skills", que medimos habitualmente con tests de preguntas de elección múltiple y respuesta única o múltiple, aún cuando algunas se refieran a casos clínicos e incluyan datos analíticos o pruebas de imagen. En relación con el mito número 2 nos dice que este tipo de examen no tiene por qué mejorar el proceso global de la evaluación en medicina, y que son muchos los estudios que demuestran que no sólo no la favorece, sino que compromete la innovación en este campo pues contraviene el principio de que la evaluación debe dirigir el aprendizaje (considérese si no el efecto deletéreo del examen MIR sobre la implementación de otras mecánicas evaluadoras en nuestras Facultades, donde los alumnos se aferran al hábito del "pass and forget" y rechazan otros tipos de evaluación limitando así gravemente su aprendizaje).

En relación con el "mito" número 3, de que el examen 
centralizado conduciría a una mayor uniformidad en los curricula, (objetivo prioritario de convergencia del EEES), la experiencia adquirida demuestra que la estandarización de los exámenes no garantiza necesariamente la homogenización de los programas docentes; por ello los países "que ha hecho ya los deberes", donde se utilizan sistemas de evaluación formativa de excelencia, rehúsan renunciar a éstos, porque hacerlo supondría un retroceso en la eficiencia y calidad de sus curricula. El "mito" número 4, de que un examen paneuropeo proporcionaría una pista de la operatividad de todos y cada uno de los programas docentes a lo largo y ancho de Europa independientemente de su diversidad, no se sustenta por estudios realizados en USA y Canadá, los cuales tampoco apoyan el mito $\mathrm{n}^{\circ} 5$ de que la aplicación del examen mejora la eficiencia de la práctica clínica beneficiando a los pacientes.

Aquí viene a colación considerar, aún sin esperar a la posible implantación de un examen paneuropeo, la conveniencia de sustituir nuestro examen MIR por otro similar al USMLE, que además de evaluar competencias clínicas, completa su tres fases coincidiendo con el final del grado, con lo cual se eliminaría el "año de preparación de la oposición MIR", adelantando el acceso del graduado a la residencia. La LOPS dictaminó en su día la obligatoriedad de implantar un MIR competencial de ámbito nacional a partir del curso 2010-11, pero todo parece indicar que este nuevo examen va a quedar postpuesto "sine die".

A las anteriores limitaciones para la realización de un examen centralizado se añadiría la dificultad logística impuesta en Europa por la diversidad existente en: 1. Las lenguas; 2. Los formatos curriculares ya operativos, o en fase de diseño; y 3. Las metodologías docentes y evaluadoras. Otros impedimentos serían el enorme tamaño de la población a examinar, y como ya se ha dicho, la oposición frontal de países con "los deberes curriculares hechos" que habiendo manifestado ya sus recelos, podrían recurrir al principio de la subsidiariedad para eludir este tipo de compromiso trasnacional. Queda así por determinar si resultaría conveniente y aplicable un examen europeo de grado, cuya adopción "tiraría hacia arriba" de los curricula de países con un retraso crónico en innovación docente, como es le nuestro, donde además tendría el efecto colateral positivo de eliminar el actual MIR que pervierte todo el proceso docente del Pregrado. A pesar de todo, no se puede excluir que si el proyecto del EEES finalmente se consolida, uno de sus pilares vaya a ser precisamente la existencia de un examen de grado paneuropeo.

\section{La situación de la convergencia con el EEES en los estu- dios de Medicina en España}

Nuestro país pertenecerá al grupo mayoritario de los que no adoptaran el esquema boloñés de dos ciclos Bache- lor y Master, y tendrán un grado de 6 años de duración con 360 ECTS. En este momento han verificado los títulos la Universidad de Navarra, y algunas creadas recientemente, como son la de Gerona, la Internacional de Cataluña, la Pompeu Fabra, la Juan Carlos I, Alfonso X el Sabio y la Europea en Madrid, y la Católica de Valencia. Además, el Consejo de Universidades ha verificado los nuevos Planes de Estudios de la Universidad de Barcelona, la Complutense de Madrid, y las de Cantabria, Extremadura, Zaragoza, Lérida y la Rovira y Virgili, que recibirán (al igual que ocurrirá con las restantes) la aprobación de las Consejerías de sus respectivas Comunidades Autónomas y el Consejo de Ministros.

Pero la cuestión nuclear no es ni la duración del grado, que podría reducirse algo como en Suecia y el Reino Unido, ni la adopción o no de los dos ciclos, sino en qué medida vamos a implantar en nuestras Facultades verdaderas reformas curriculares que incluyan: A. La definición explícita de los objetivos y las metodologías docentes y evaluadoras; B. La integración en mayor o menor grado las enseñanzas básicas y clínicas, organizando el aprendizaje alrededor de módulos, bloques o ciclos vitales; C. La utilización de alguna de las variantes para la resolución de problemas; D. Una enseñanza práctica centrada en el paciente tanto real como virtual, que conlleve un contacto precoz con el enfermo y la inmersión prolongada en el contexto clínico, tanto a nivel hospitalario como en el ámbito de la medicina primaria, que aseguren la adquisición de competencias clínicas que deberán ser evaluadas de manera objetiva y estructurada en un proceso repetitivo a lo largo del grado; y E. Propiciar la iniciativa y autonomía del alumno para guiar su propio aprendizaje, enseñándole a "aprender por sí mismo" (autoaprendizaje), y adquirir una mentalidad crítica junto con el nuevo hábito de estudio que resulta más necesario en medicina que en ninguna otra carrera ("longlife learning"), utilizando para ello de manera rutinaria los nuevos recursos tecnológicos ("e-learning").

$\mathrm{Si}$ se tiene en cuenta el retardo en la implantación de innovaciones curriculares en nuestras Facultades, las carencias y obstáculos de nuestra situación de partida, y la necesidad de elaborar los nuevos Planes de Estudio de manera precipitada, se corre el riesgo de improvisar para salir del paso, y caer en la tentación de aplicar simples retoques cosméticos a lo que ya tenemos, en vez de introducir verdaderos cambios curriculares. Y es que en algunas Facultades puede ocurrir que la elaboración del nuevo Plan de Estudios sea asumida por unos pocos, que tras improvisar un diseño más o menos "apañado" convirtiendo los módulos en las clásicas asignaturas y computando los viejos créditos por los nuevos ECTS, logren pasarlo para su aprobación en las correspondientes Juntas de Facultad sin el necesario debate previo con el profesorado y el alumnado. Así, el resultado final podría ser que no se cambiase 
nada verdaderamente esencial en la estructura curricular previa, con lo cual nuestro retraso en relación con los países más avanzados no sólo no se reduciría, sino que aumentaría más cada vez.

Habrá que esperar para comprobar qué "dosis" de cambio curricular efectivo aplicará cada Facultad, pero las manifestaciones de nuestros Decanos sobre la "adaptación a Bolonia" no permiten ser muy optimistas, y es que en su conjunto traslucen escepticismo, pesimismo, y quizás una cierta resignación ante lo que se considera ya como un fracaso anunciado.

\section{Las opiniones de los decanos de las Facultades españolas sobre el plan de Bolonia}

El análisis de las opiniones de los decanos "acerca de la implantación del Plan de Bolonia en Medicina" recogidas por Diario Médico² ("Bolonia: los mismos medios, menos libertad de cátedra y más burocracia” 21 Abril, 2009) revela bien la incertidumbre que acecha a la implantación de curricula modernizados en nuestras Facultades. De entrada los decanos denuncian con razón que "Bolonia nos ha sido impuesta", y que "estamos ante un proyecto en el que ni profesores ni alumnos hemos participado". Esta falta de debate entre los agentes implicados y de una acción colegiada para abordar la creación de los nuevos Planes de Estudio contrasta claramente, por poner algunos ejemplos tan solo, con la metodología aplicada en el Reino Unido para diseñar los nuevos planes de estudio (ver por ejemplo el documento "The Scottish Doctor"), o el más reciente Foundation Programme, que articula la transición de los nuevos estudios de grado al postgrado, en el que estuvieron directamente involucrados durante largos periodos de tiempo todos los agentes implicados (diversos "stakeholders" o representantes del Sistema Nacional de Salud, Universidades y Decanatos, Reales Colegios, Sociedades Científicas, etc).

Entre las múltiples adversidades que amenazan la consecución del necesario cambio curricular, nuestros decanos destacan: 1. La falta de medios económicos para una enseñanza más individualizada; 2 . La menor libertad de cátedra; 3 . El incremento de la burocracia; 4. La improvisación legislativa; y 5. La falta de suficientes profesores clínicos acreditados. Aunque estos impedimentos se dan en realidad, es conveniente matizar qué hay de verdad, y qué de relativamente cierto en relación con cada uno de ellos, más aún cuando tomados en su conjunto pudieran ser utilizados como coartada para perpetuarse en el estancamiento docente.

Que ha habido improvisación legislativa es palmario. Ya se ha comentado que las normativas europeas han cristalizado en regulaciones impuestas a plazo fijo desde los ministerios de los diferentes países que actúan como polea de transmisión para traspasarlas a la "trinchera docente". En este proceso, efectivamente sobrecargado de burocracia inútil, siempre se encuentran colaboracionistas académicos capaces de realizar el "prodigio" de "adaptar la propuesta boloñesa al terreno" sin cambiar en realidad nada esencial.

Es innegable que la falta de medios económicos es capaz por sí sola de dar al traste con cualquier proceso de mejora curricular, y cuando uno examina algunos recientes convenios entre Universidades y Consejerías de Sanidad se puede leer en ellos que "las mejoras docentes se llevaran a cabo con los recursos ya disponibles", cuando es bien sabido que nada se cambia a mejor "a coste cero". Dejando a un lado las necesidades de los departamentos de ciencias básicas (profesorado, laboratorios, etc), y limitándonos a la docencia de la práctica clínica, resulta obvio que para implantar una enseñanza "más individualizada" que contemple además verdaderas tutorías sería necesario integrar en la tarea docente a la mayoría, si no a todos, los profesionales que trabajan a nivel intra y extrahospitalario en las instituciones sanitarias implicadas en la enseñanza del Pregrado, y que esa nueva reorganización y aprovechamiento de la "fuerza docente" conlleva inevitablemente un incremento de los costes, al igual que ocurre, por poner otro ejemplo, con la incorporación de los recursos tecnológicos que hacen posible la aplicación de nuevas técnicas docentes de simulación y facilitación del autoaprendizaje.

En relación con el "atentado del Plan de Bolonia contra la libertad de cátedra", es preciso reconocer que con frecuencia esta libertad se utiliza para enrocarse en dinámicas docentes acartonadas e inoperantes, y que mientras no se use para mejorar la enseñanza impulsando y sosteniendo la renovación curricular, no habría porqué lamentar su limitación, o la imposición de cambios en la manera en que es ejercida actualmente. Y es que para progresar en innovación docente parece imprescindible disponer en nuestro ámbito de un nuevo marco administrativo, o de instrumentos que controlen los efectos negativos del corporativismo que favorece la endogamia, por un lado, y del ejercicio del individualismo excluyente por parte de profesores situados en posiciones administrativamente determinantes, por otro. Este objetivo podría alcanzarse creando Unidades Docentes integradas por profesores interesados en la docencia moderna, que ostentasen por delegación de los propios departamentos "la libertad necesaria" para coordinar y acordar el diseño de un buen currículum, monitorizando después su desarrollo, y cuyas determinaciones quedasen siempre a cubierto de posicionamientos arbitrarios de profesores o departamentos.

Pero de esta opción no hablan los decanos, y ello es de lamentar porque de haber dispuesto de Unidades Docentes, tendríamos ahora la "masa crítica" de expertos en docencia médica necesaria para dirigir la creación de un esquema básico o componente nuclear de un nuevo Plan de Estudios 
que podría compartirse entre las diferentes Facultades. En nuestro país los expertos en docencia son pocos y además trabajan en solitario, y esta carencia, reveladora como pocas del origen de nuestro atraso curricular, puede convertirnos más en responsables que en víctimas del posible fracaso en la modernización de los programas docentes que exige este momento histórico.

Igualmente, la mayoría de los decanos reiteran que "no hay suficientes profesores clínicos acreditados (titulares y catedráticos) para abordar la enseñanza mucho más práctica e individualizada" que exige Bolonia. Sin embargo, esta afirmación debe ser matizada también. Aunque es cierto que en algunas Facultades el número de profesores disponibles para llevar a cabo una buena enseñanza práctica es tan insuficiente que haría fracasar el mejor Plan de Estudios, en otras ese número resulta suficiente para abordarla con garantía, siempre que los implicados cumplan su compromiso docente. Sin embargo, la realidad es que bastantes profesores no sólo no la practican, sino que tampoco entra en sus planes de futuro hacerlo, y son todavía mayoría los que siguen creyendo que impartir lecciones y preparar los correspondientes exámenes teóricos es su principal, si no el único y más excelso, cometido docente. En relación con este punto, y aunque la pregunta parezca quimérica ¿No debería aprovecharse la ocasión histórica de cambio que representa la adaptación al EEES, para "recomponer", insertándolo en el contexto de un nuevo enfoque curricular, todo el sistema de contratación y promoción del profesorado, redistribuyendo las contraprestaciones económicas y curriculares disponibles y garantizando a un tiempo su formación docente y el control de calidad de su activad?

En suma, nuestros decanos identifican las "asechanzas" que amenazan la renovación curricular en nuestras Facultades, pero marginados como están en el desarrollo del proceso boloñés, no aciertan a proponer una salida colegiada que permita introducir las innovaciones educativas recomendadas desde el EEES. Por eso, la afirmación del portavoz de la Conferencia Nacional de Decanos de que "las Facultades de Medicina ya estamos preparadas para el cambio", resulta cuestionable.

¿En qué consiste estar preparado para el cambio? ¿No hemos convenido en que no ha habido el debate necesario para alcanzar esa preparación, y en que la gran mayoría del profesorado y el alumnado ignora el perfil y la estructura de los nuevos curricula, las teorías del aprendizaje del adulto, en qué consiste el movimiento competencial, o cúales son y cómo se aplican las nuevas técnicas de evaluación clínica objetiva estructurada? ¿Acaso están los profesores adecuadamente preparados y motivados para abordar la integración de las enseñanzas básicas y clínicas, para aplicar la técnica de la resolución de problemas en cualquiera de sus variantes, o para el uso de pacientes virtuales y simuladores? ¿Es consciente el profesorado de que la eva- luación debe dirigir el aprendizaje y de la trascendencia que tiene enseñar al alumno a aprender? ¿Saben los profesores, o les importa siquiera, cómo se evalúa el funcionamiento de un programa docente? ¿No habla el propio Presidente de "la desmotivación y el cansancio crónico del profesorado", o de "la resistencia numantina de una parte de los profesores que son enemigos de cualquier cambio", y que según él mismo "han demostrado últimamente que no tienen ninguna intención de adaptarse a las nuevas formas poniendo en peligro la viabilidad de todo el proceso"? La pregunta final podría ser: ¿No están acaso nuestras Facultades cautivas de la negligencia y apatía de su propio profesorado? Miguel de Unamuno en su libro De la enseñanza superior en España, aporta el siguiente comentario que sigue siendo perfectamente actual: "No hace falta pedir al ministro que varie el plan de estudios mediante la "Gaceta"; mientras los que hayan de explicar las asignaturas nuevas o renovadas no varien el plan de su espíritu o no sean otros, la variación será peor que nula, pretexto de vagancia, mientras que si se variase, los que han de enseñar y tuvieran fe en la enseñanza y espiritu cientifico y amplitud de alma harian bueno cualquier plan (...). Hay que crear fe, fe verdadera en la enseñanza, y sólo se crea esta fe enseñando con ella, con fe viva (...)”.

Que la preparación para el cambio de paradigma docente que preconiza el EEES deja mucho que desear en nuestras Facultades, queda claro si constatamos las siguientes limitaciones y carencias en la mayoría de ellas: 1 . Cuerpos profesorales envejecidos e ignorantes de la estructura y dinámica docente de los nuevos curricula a quienes su Facultad no rescata aplicando algún sistema de formación que mejore su visión y desempeño docentes; 2 . La adherencia a una rutina docente obsoleta basada fundamentalmente en la transmisión de datos, y en una metodología evaluadora limitada en la mayoría de los casos a medir la memorización de los mismos por el alumno, que es impulsado así a seguir la estrategia del "pasa y olvida", reforzada en nuestro país por el omnipresente examen MIR; 3. La falta de integración de las enseñanzas básico-clínicas y del uso de metodologías docentes que la propicien; 4. La crónica distinción entre docentes y "no docentes" en los hospitales universitarios, que si bien puede bastar para practicar una enseñanza predominantemente teórica, impide aplicar cualquier programa de enseñanza práctica de calidad; 5 . La precariedad de la enseñanza práctica, que en el mejor de los casos se imparte con estrategias deslabazadas que no utilizan los nuevos instrumentos de evaluación capaces de medir de manera objetiva y estructurada el nivel de competencia clínica alcanzado por el alumno en las sucesivas etapas de su paso por los cursos clínicos; 6. La tardanza en la exposición de los alumnos al contexto clínico y su "secuestro" en el hospital, que les impide acceder a ese conocimiento del espectro completo y el verdadero perfil 
de las enfermedades más comunes que sí proporciona en cambio la rotación sistemática por atención primaria; 7 . El retraso en la utilización de los recursos electrónicos favorecido además por la marginación de la lengua inglesa en todas las etapas del aprendizaje; 8. La rígida estructura departamental que dificulta la integración vertical y horizontal de las enseñanzas, y retroalimenta por su propia dinámica la incomunicación y un inmovilismo recelosamente defensivo del profesorado que para nada favorecen la creación de Unidades Docentes o Comités Curriculares; 9. La falta de continuidad y ensamblaje entre las enseñanzas del Pregrado y el Postgrado; y 10. Los desajustes del actual sistema de acceso y promoción en la carrera académica.

Acabaremos diciendo que a pesar de los malos augurios derivados de las imperfecciones del proceso boloñés y las deficiencias crónicas de nuestro particular ambiente docente, siempre existe (con Bolonia o sin Bolonia) la posibilidad de incorporar a nuestros Planes de Estudio las metodologías docentes y evaluadoras que fecundan los nuevos curricula. Pero no hay que perder de vista que el curriculum es tan sólo un marco, y que ningún tipo de estructura curricular al uso, incluida la aplicada en la mayoría de las Facultades españolas que conserva la anticuada separación de las materias básicas y clínicas, impide de manera definitiva la incorporación de los ingredientes que definen la verdadera innovación docente. Que esto es así lo han demostrado los cuerpos docentes de muchas escuelas de medicina europeas que han actualizado sus curricula al margen, más que al compás, del proceso de Bolonia.

\section{Bibliografía}

1. Archer, J.C.: European licensing examinations. The only way forward. Medical Teacher 2009; 31: 215-216.

2. Bolonia: los mismos medios, menos libertad de cátedra y más burocracia. Diario Médico. Abril, 21, 2009.

3. Christensen, L.: The Bologna Process and medical education. Medical Teacher 2004; 26: 625-629.
4. Fernández Liria, C., Serrano García, C.: El Plan de Bolonia. Libros de la Catarata, Madrid, 2009.

5. García Manjón, J.V.: Hacia el Espacio Europeo de Educación Superior. El reto de la adaptación de la Universidad a Bolonia. Netbiblo, La Coruña, 2009.

6. Harden, R.: Five myths and the case against a European or national licensing examination. Medical Teacher 2009; 31: 217-220.

7. Humphrey, H.J., Levinson, D., Smith, L.: The Medical School on the University Campus: 20th-Century Legacy and 21st-Century Aspirations. Acad Med 2010; 85: 273-282.

8. Lopez Herrerías, J.A.: La Universidad y el proyecto de Bolonia: EEES. Hergué Editorial, Huelva, 2008.

9. Melnick, D.E.: Licensing examinations in North America; Is external audit valuable? Medical Teacher 2009; 31: 212-214.

10. Patricio, M., Engelsen, C.D., Tseng, D., ten Cate, O.: Implementation of the Bologna two-cycle system in medical education: Where do we stand in 2007? Results of an AMEE-MEDINE survey. Medical Teacher 2008; 30: 597-605.

11. Sosa Wagner, F.: El mito de la Autonomía Universitaria. Aranzadi Editorial, Pamplona, 2007.

12. van der Vleuten, C.P.M.: National, European licensing examinations or none at all. Medical Teacher 2009; 31: 189191.

13. van Scharvendijk, Ch.F.H., Miecka, J.: Harmonization of the bachelor-master system in the curricula of the medical doctor and the biomedical sciences. Report on a 2 days workshop. Medical Teacher 2007; 29: 267-269.

Lobato, R.D.; Lagares, A.; Alén, J.F.; Alday, R.: El desarrollo del proceso de "Bolonia" y el Grado de Medicina. Situación actual y expectativas para su implantación definitiva. Neurocirugía 2010; 21: 146-156.

Correspondencia postal: Ramiro D. Lobato. Servicio de Neurocirugía. Hospital 12 de Octubre. Avda de Córdoba s/n. 28041 Madrid. 